\title{
A Study on Localization in Wireless Sensor Network Using Neural Network
}

\author{
K. Madhumathi ${ }^{1}$ and T. Suresh ${ }^{2}$ \\ ${ }^{1 \& 2}$ Assistant Professor, \\ ${ }^{1}$ Department of Bachelor of Computer Applications, Anna Adarsh College for Women, Chennai, Tamil Nadu, India \\ ${ }^{2}$ Department of Computer Science and Engineering, Annamalai University, Chidambaram, Tamil Nadu, India \\ E-Mail: madhumathi@hotmail.com, sureshaucse@gmail.com
}

\begin{abstract}
Wireless Sensor Network consists of large number of autonomous, small, low cost sensor nodes that are spatially distributed in areas of investigation like disaster management, Military, environmental monitoring etc. The main purpose of using those nodes is to collect information from source and process it in destination. But the data received in the destination are useless unless the exact location of source is not known. The task of finding physical coordinates of these sensor nodes in WSNs area is known as localization. One solution for the above problem is manual configuration of sensor but it is unfeasible if the area of deployment is large or inaccessible. Therefore, we use localization techniques which help to capture the location of nodes in wireless sensor network. This study analyses localization algorithms with their pros and cons.

Keywords: Wireless Sensor Network, Localization, Localization Algorithms, Neural Networks
\end{abstract}

\section{INTRODUCTION}

A WSN is a technology which consists of large number of sensor nodes that gathers information from large geographical areas and routes the observed data using some routing protocol over wireless channel to Gateway (Base Station or Sink Node) via sensor nodes. Further they are transferred to remote client either via wired or wireless through internet.

\section{CONCEPT OF LOCALIZATION}

Localization is a procedure to find the geological location of every single sensor node in the Sensor field. Localization concept as shown in Fig.1

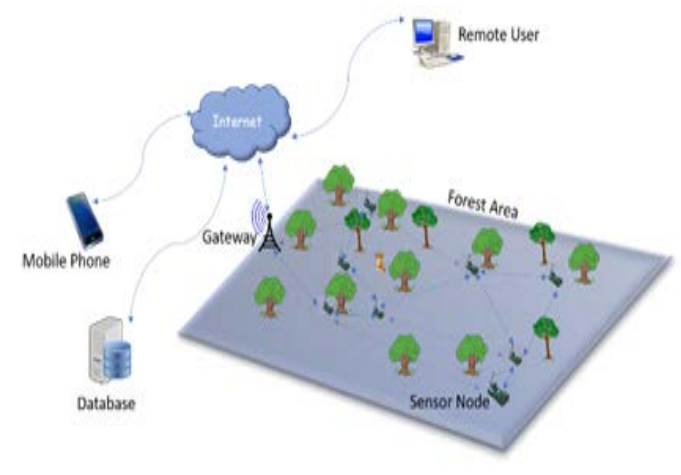

Fig. 1 Concept of Localization
Since the data received is useless without the location information (from where it is sent). Example: Localization is the main requirement of WSN. Consider a forest fire, to know the exact location of the fire; it is essential to know the precise location of sensor node which sensed that information. One approach is Manual Configuration of all the sensor nodes, but it is improbable if the WSN area is large. The other way is by installing GPS equipped sensor device [2]. The advantage of this method is that it gives accurate position information and high anti-interference. The flaws are high energy consumption, cost.

To overcome the above disadvantage few neighbouring sensor nodes are equipped with GPS.

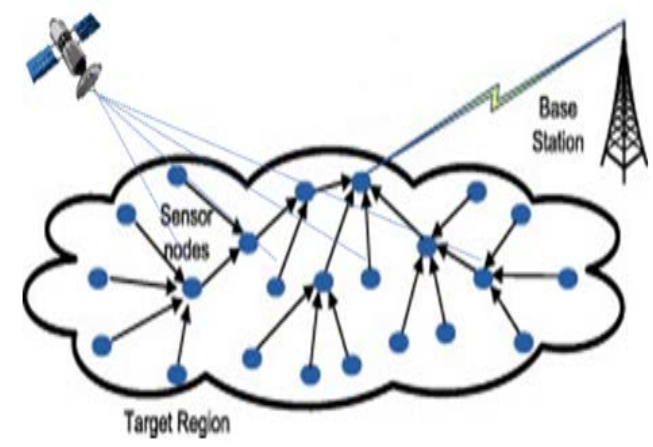

Fig. 2 Few Nodes enabled with GPS

Other nodes (Non-Beacon nodes or Blind nodes) are made to identify their locations with the help of those nodes whose locations are already known (beacon or anchor nodes) as shown in Fig 2.This paper concentrates on the localization techniques used by the sensor nodes to identify their location.

A. Localization Overview: A localization algorithm localizes nodes based on input data. If there is a beacon node in the sensor area, then location of anchornode is the input to it and the output of localization algorithm for it is absolute coordinate. Network without anchor node can estimate their location by using distance or angle between nodes and the output of these anchor free methods is relative coordinate. Localization consists of 3 stages as shown in Fig 3.

1. Distance/Angle estimation between the nodes, 
2. Position calculation of a single node,

3. Localization algorithm - used for localization of whole network.

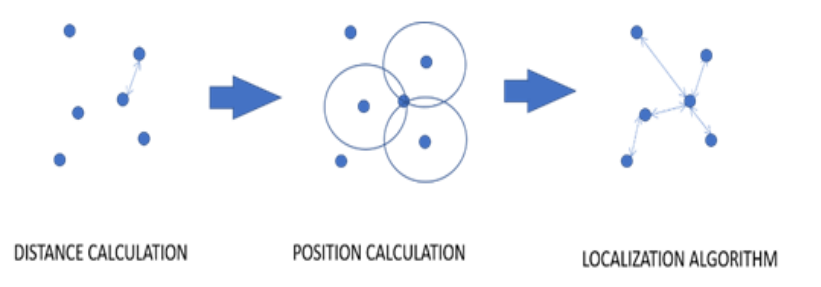

Fig. 3 Three Stages of Localization

\section{B. Localization Stages \\ 1. Distance/Angle Estimation}

a. Lateration: The signals transmitted from the special antenna in each sensor node helps to estimate distance between nodes. The main disadvantage is more energy is needed and there is an increase in hardware cost. Different techniques used are:

Time of Arrival (ToA): The propagation time of the signal between source and destination nodes are measured in view of the speed of signal propagation. Given this distance, the localization is performed.

Time Difference of Arrival (TDoA): This method uses different signals like radio frequency, ultrasound signal of different velocity to calculate the distance. TDoA method does not need the synchronization of transmitter and receiver. Received Signal Strength Indication (RSSI): Receiver's Received Signal Strength Indicator circuit estimates the distance based on the received signal strength. Radio signal attenuates as distance increase [3]; therefore, it can be applied to line of sight networks only.

b. Angulation: Angle of Arrival (AoA): This method calculates distance by using the angle to localize the target. Here there will be a group of antennas to estimate the direction of received signal. Line-of-Sight and antenna configuration plays a vital role here.

\section{Position Calculation of a Single Node}

a. Trilateration: Three Anchor nodes are used to find the location of blind node. It uses distance between anchor nodes and unknown nodes.

b. Multilateration: This technique uses more than three anchor nodes to find the location of blind node.

c. Triangulation: It uses at least two angles of a blind node from two anchor nodes to estimate the location of blind node.

\section{Localization Classification}

a. Traditional Methods

i. GPS Based and GPS Free

a) GPS based: Best method for outdoor Localization, all nodes are equipped with GPS based b) GPS Free: Some Nodes need to be GPS based and other nodes location can be calculated using the distance between the node relative to its local network.

\section{ii. Centralized and Distributed}

a) Centralized: Entire information is passed to one node (Central Base Station), which computes the position of nodes and sends it back to the requested node.

b) Distributed: Sensor calculates its positions individually and communicates directly with anchor node.

iii. Stationary and Mobile Sensor Nodes

a) Stationary: Sensor are static in nature (i.e.) they are fixed at one place.

b) Mobile: sensor nodes are free to move independently in any direction, and will therefore change its links to other nodes frequently.

\section{iv. Range Based and Range Free}

a) Range Based: It uses distance and angle, RSSI information from anchor node to calculate location and needs additional device to find location. They are more sensitive to noise and it fades.

b) Range Free: It uses GPS or artificially deployed information to acquire its location. Prone to position estimation errors.

\section{v. Anchor Based and Anchor Free}

a) Anchor Based: Few nodes positions are known; Blind nodes' position is calculated using known nodes location.

b) Anchor Free: It calculates relative position of nodes instead of absolute position.

\section{b. Localization Algorithms}

i. Centroid uses centroid- Mathematical concept. By counting number of signals receiver from nearby anchor nodes and by calculating the centroid we find the locations of non-beacon nodes.

ii. DV-HOP uses hop count from beacon to non-beacon nodes and average distance per hop as parameter to find blind nodes location.

iii. Appropriate point in triangle (APIT) [4] a sensor node selects three beacon nodes and finds whether it is inside or outside by combining those anchors by constructing a triangle. Process is repeated for many times for different combination. Finally, the COG (Center of Gravity) is calculated using all intersection of triangles in which the target node is found.

iv. Distributed Location Estimating Algorithm (DLE) [5], here estimative rectangle (ER) is computed using the location information of neighbouring anchor nodes to find the location of blind nodes. 
$v$. Distributed range free localization scheme (DRLS), here non-beacon nodes uses Beacon nodes which are within two hop distance from them. Grid scanning algorithm is used to find the preliminary position of a normal node and accuracy is improved by using vector-based refinement method.

vi. MDS-MAP [6]: This method does not need anchor or beacon nodes to start with. It builds a relative map of the nodes even without anchor nodes and next with three or more anchor nodes; the relative map is transformed into absolute coordinates.

In traditional method, the parameters related to angle and distance of arriving signal is accuracy however, they require a dedicated hardware, which are both expensive and energy consuming. When distance measurement is noisy then triangulation, trilateration method, Multilateration method does not give the accurate positions of the nodes and become useless in traditional method.

TABLE I VARIOUS CATEgORIES OF TRADITIONAL METHODS

\begin{tabular}{|l|c|c|c|c|}
\hline \multicolumn{1}{|c|}{ Category } & Location Finding & Power Consumption & $\begin{array}{c}\text { Hardware } \\
\text { Cost }\end{array}$ & Algorithms \\
\hline GPS Based & Accurate & high & High & Weighted Least Square Method \\
\hline GPS Free & Less Accurate & Medium & Less & MSPA \\
\hline Centralized & Accurate & High & Less & MDS - MAP, SDP \\
\hline Distributed & Less Accurate & Low & High & APIT, APS, REP, DLE, DRSL \\
\hline Range Based & Less Accurate & High & Very less & TDoA, DoA, RSSI \\
\hline Range Free & Less Accurate & Medium & Very Less & APIT, DRSL, DLE, Centroid, MDS \\
\hline Anchor Based & Accurate & High & High & N - HOP, Multi Literation \\
\hline Anchor Free & Less Accurate & Low & High & AFL, AFLR \\
\hline
\end{tabular}

\section{c. Computational Intelligence Methods}

A neural network is a group of algorithms which diagnose original relationships in a set of data through a process that imitators the working of human brain. The interconnections between nodes "neurons" have activation functions. By varying the activation functions, the network generates the best possible result without needing to redesign the output criteria.

\section{Need of Artificial Neural Network}

The benefits of using the computational intelligence in Wireless Sensor Network's Localization process is that prior knowledge of the noise distributions is not required. Noisy distance measurements can be used directly to train the network with actual coordinate locations. There are mainly three types of Neural Network used for localization [7].

a. Feed forward Neural Network - Artificial Neuron: It is the simplest network in which, the data passes through the input node and exit on to output node in one direction, with or without hidden layer. Feed forward network without hidden layers are called single layer perceptron (SLP). Feed forward layer with hidden layers are called multilayer perceptron (MLP)

b. Radial Basis Function Neural Network (RBF): Radial basic functions consider the distance of a point with respect to the center. It has input layer, a hidden layer and an output layer. The hidden layer uses Gaussian transfer functions which are used as an activation function for nodes. c. Recurrent Neural Network (RNN): RNN saves the output from a layer and feeds it back as input to find the result. It is similar to MLP with feedback concept.

\section{EXPERIMENT APPROACH}

In WSN using Neural network consists of three major phase. The first phase represents Deployment. After deployment represents Collecting Dataset and finally deals Training ANN

\section{Deployment $\rightarrow$ Collecting Dataset

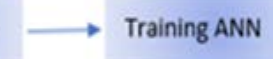

Fig. 4 Major phases of Experimental approach using WSN

A. Deployment of WSN: Consider an environment which has five beacon nodes and several non- beacon nodes. The anchor nodes are places in fixed predefined position. Training data and test data are collected. Training data is important since neural network keeps on varying weight until it achieves the target output.

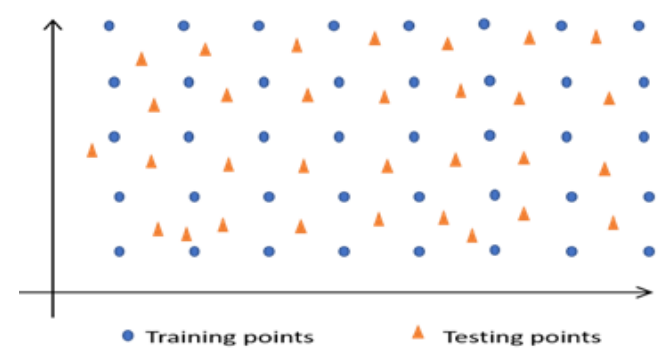

Fig. 5 Location of Training and Testing Points 
B. Collecting of Training Data: The mobile nodes are place in between five beacon nodes and training data are collected, by finding the distance between the mobile node and beacon nodes. Therefore, the input to the neural network is the distance between mobile nodes and beacon nodes or RSSI signal [8] and output will be the location of the mobile node. To get more data on distance information above process is repeated many times. Every time the value may vary due to noise in the system. This procedure will help in finding the noise in the system. By training our neural network with those data accuracy also increases.

C. Training ANN: The network can be trained in MATLAB. [9]. The training function like trainlm (LevenbergMarquardt algorithm: Fastest training algorithm for networks of moderate size, has memory reduction feature for use when the training set is large). Traingdx (Adaptive learning rate. Faster training than traingd, but can only be used in batch mode training), trainbr (Bayesian regularization. Modification of the Levenberg-Marquardt training algorithm) can be used. Learning functions like learned Gradient descent weight/bias learning function, learngdm Gradient descent w/momentum weight/bias learning function, learnp Perceptron weight/bias learning function can be used.

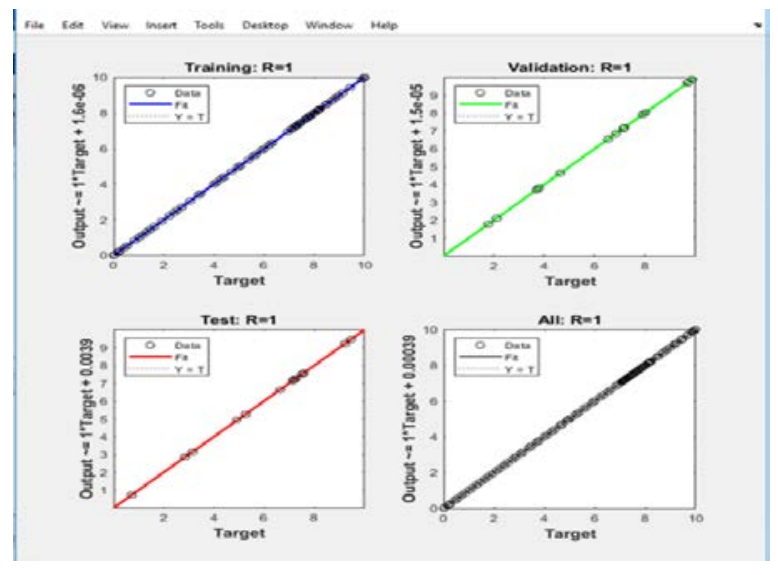

Fig. 6 Regression Plot for Localization

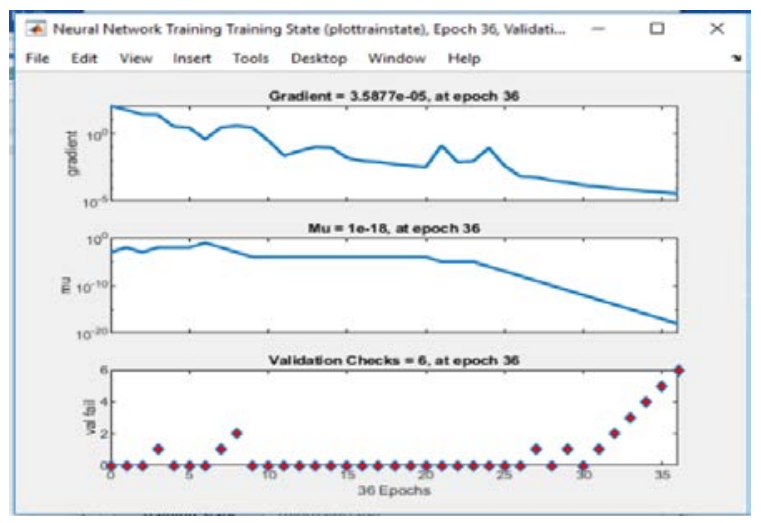

Fig. 7 Training State plot for Localization
Regression plot is used to validate network performance. This regression plot displays the network output with respect to the target for training, validation and test set. Training State Plot: Gradients are the values of back propagation gradients on each iteration.

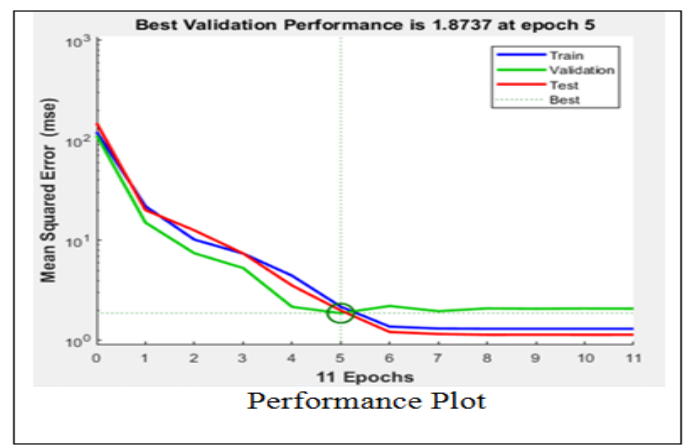

Fig. 8 Best Performance plot for Localization

\section{CONCLUSION}

It was found that RNN is prone to high percentage of Distance error estimation when compared with RBF and RNN. RBF has best localization performance [10]. MLP uses least memory and has low computational cost with compared with RBF and RNN which is its big advantage. When using regression plot MLP fits best than RNN. Therefore, MLP performs best in Localization in wireless sensor Network when compared with RNN and RBF.

\section{REFERENCES}

[1] Akyildiz W. Su, Y. Sankarasubra maniam, and E. Cayirci, "Wireless sensor networks: A survey,” Computer Networks (Elsevier) Journal, Vol. 38, No. 4, pp. 393-422, Mar. 2002.

[2] Bo Cheng, Rong Du, and Bo Yang, "An Accurate GPS-based Localization in Wireless Sensor Networks: A GM-WLS Method," International Conference on Parallel Processing Workshops, 2011.

[3] S. N Radhika, and K. V. Chaitra, "RSSI based Localization in WSN" International journal of Computer science and Applications, Vol. 12, Jan. 2018

[4] Rakhi Sharma and Sona Malhotra, "Approximate Point in Triangulation (APIT) based Localization Algorithm in Wireless Sensor Network" International Journal for Innovative Research in Science \& Technology, 2015

[5] Jang-Ping, and Chih-Shun Hsu, "A Distributed Location Estimating Algorithm for Wireless Sensor Networks "Proceedings of the IEEE International Conference on Sensor Networks, Ubiquitous, and Trustworthy Computing (SUTC’06), 2006

[6] Lalita Baburao Choudhary, and V.V. Yerigeri "MDS- MAP Algorithm for Localization", International Journal of Advance Engineering and Research Development, Vol. 5, No. 3, Mar2018

[7] Mohammad Shaifur Rahman, Youngil Park, and Ki-Doo Kim, "Localization of Wireless sensor Network Using Artificial Neural Network," Proc. 9th International Conference on Communications and Information Technologies, 2009

[8] Vikram Sonmore, Vaibhav Bagal,Himanshu Sachdeva, and Tanmay Choudhari, "Indoor 3D Localization of WSN using Neural network", IRJET Vol. 5, Apr 2018.

[9] Swat Singhal, K. R.Santosh. Bharti, and Garuma Kaushik, "Localization in Wireless Sensor network: An optimistic Approach", IJCSIT, Vol. 4, No. 2, pp. 224 -226, 2013

[10] Ali Shareef, Yifeng Zhu, and Mohamad Musavi, "Localization Using Neural Networks in Wireless Sensor Networks" Mobilware'08, Feb 12-15, 2008. 